\title{
An innovation diffusion model of a local electricity network that is influenced by internal and external factors
}

Article

Accepted Version

Creative Commons: Attribution-Noncommercial-No Derivative Works 4.0

Hattam, L. and Vukadinovic Greetham, D. (2018) An innovation diffusion model of a local electricity network that is influenced by internal and external factors. Physica A:

Statistical Mechanics and its Applications, 490. pp. 353-365. ISSN 0378-4371 doi:

https://doi.org/10.1016/j.physa.2017.08.014 Available at https://centaur.reading.ac.uk/72067/

It is advisable to refer to the publisher's version if you intend to cite from the work. See Guidance on citing.

To link to this article DOI: http://dx.doi.org/10.1016/j.physa.2017.08.014

Publisher: Elsevier

All outputs in CentAUR are protected by Intellectual Property Rights law, including copyright law. Copyright and IPR is retained by the creators or other copyright holders. Terms and conditions for use of this material are defined in the End User Agreement. 


\section{CentAUR}

Central Archive at the University of Reading

Reading's research outputs online 


\title{
An innovation diffusion model of a local electricity network that is influenced by internal and external factors
}

\author{
Laura Hattam and Danica Vukadinović Greetham*
}

\begin{abstract}
Haynes et al. (1977) derived a nonlinear differential equation to determine the spread of innovations within a social network across space and time. This model depends upon the imitators and the innovators within the social system, where the imitators respond to internal influences, whilst the innovators react to external factors. Here, this differential equation is applied to simulate the uptake of a low-carbon technology (LCT) within a real local electricity network that is situated in the UK. This network comprises of many households that are assigned to certain feeders. Firstly, travelling wave solutions of Haynes' model are used to predict adoption times as a function of the imitation and innovation influences. Then, the grid that represents the electricity network is created so that the finite element method (FEM) can be implemented. Next, innovation diffusion is modelled with Haynes' equation and the FEM, where varying magnitudes of the internal and external pressures are imposed. Consequently, the impact of these model parameters is investigated. Moreover, LCT adoption trajectories at fixed feeder locations are calculated, which give a macroscopic understanding of the uptake behaviour at specific network sites. Lastly, the adoption of LCTs at a household level is examined, where microscopic and macroscopic approaches are combined.
\end{abstract}

\section{Introduction}

Rogers (1962) proposed that the diffusion of a new technology through a social network resulted from the social interactions of its members. Bass (1969) built upon this work by applying a nonlinear ordinary differential equation to predict innovation diffusion as a function of time, which contained parameters associated with the 'innovators' and 'imitators' within the network. From this, the 'S-curve' solution that predicts the number of adopters with time was derived. Later, Haynes et al. (1977) extended the Bass model to also include spatial dependence, which is of the form

$$
\frac{\partial N}{\partial t}=(p+q N)(1-N)+k\left(\frac{\partial^{2} N}{\partial x^{2}}+\frac{\partial^{2} N}{\partial y^{2}}\right),
$$

where $x$ and $y$ are spatial variables, $t$ is time, $N(x, y, t) \in[0,1]$ represents the percentage of innovation adopters at the location $(x, y)$ and time $t, k$ is the diffusion coefficient, and $p$ and $q$ are the innovation and imitation coefficients respectively. The innovation parameter relates to external influences, such as advertising

${ }^{*}$ Department of Mathematics and Statistics, University of Reading, UK 
and the imitation coefficient corresponds to internal effects, like word-of-mouth. After some manipulation of (1), it becomes the Fisher equation, which was used by Fisher (1937) to model the spread of a mutant gene.

Here, (1) is applied to determine the uptake of low-carbon technologies (LCTs) within a sample area. In particular, our focus is the implementation of photovoltaics (PVs) across the UK. Aiming to reduce the country's carbon footprint by 2020 and beyond, the UK government plans to encourage and facilitate PV installations ${ }^{1}$. As well, they seek to take advantage of the public's $85 \%$ approval rating of PVs, which suggests that this technology is the most popular LCT within the $\mathrm{UK}^{1}$. Therefore, projecting a monotonically increasing number of PV ownership over time is a feasible scenario here and Haynes' equation, (1), is an appropriate model for such a process. Moreover, (1) takes into account the influence of government initiatives and public perceptions with $p$ and $q$ respectively, which allows us to simulate the growth of very localised innovation clusters. This is a known adoption pattern for PVs, which was demonstrated by Snape (2016), where it was revealed that PV clusters have formed spatially across the UK.

It should be noted that the technology will be pervasive when applying Haynes' model, which is a reasonable assumption within the context considered here. However, other approaches could be utilised if this is an unrealistic prospect or if adoption populations are expected to regress at some point. For example, Masini and Frankl (2003) and Michalakelis and Sphicopoulos (2012) applied stochastic models for determining innovation diffusion. Although the subsequent results can be more difficult to analyse, this model type can sometimes explain behaviour that is not captured by a purely deterministic technique, as discussed by Constable et al. (2016). Whilst our study uses a deterministic method that can be solved analytically and numerically, the stochastic component is later incorporated into our model at a household level. This then gives greater flexibility at a higher resolution to explore technology uptake.

Morrill (1970) described the innovation adoption process as a wave-like phenomenon propagating in space and time, using such physical examples as the implementation of tuberculosis controls within agricultural regions to demonstrate this. Therefore, given that (1) transforms into the Fisher equation, known travelling wave solutions of this equation could be used to predict the spread of innovations across certain geographies. Tyson and Brazhnik (2000) derived travelling wave solutions to the Fisher equation over two spatial dimensions. In particular, they defined the well-known Fisher travelling wave identified by Ablowitz and Zeppetella (1979) in terms of two spatial variables. This solution is of the same form as the S-curve, although it varies with space and time, and is used here to predict the adoption behaviour.

Shinohara and Okuda (2009) and Karakaya (2016) used differential equations to model the spread of LCTs. They both studied geographic areas with complicated geometries and therefore, the finite element method (FEM) was utilised. Our area of interest is also complex and so the FEM will be applied here, where a real local electricity network is examined with 9484 households and 249 feeders. A group of households are connected to the network via a feeder, and therefore, a feeder corresponds to a spatial clustering of homes. The aim is to obtain S-curves at these network locations, which will be projections for the number of household adoptions along a particular feeder. Currently, accurate values for the parameters $p$ and $q$ for the area under examination are unknown. However, over the coming years, further information about the uptake behaviour throughout the UK will presumably become available. As a result, $p$ and $q$ can then be obtained

\footnotetext{
${ }^{1}$ Department of Energy and Climate Change, 2014, https://www.gov.uk/government/uploads/system/uploads/attachment_ data/file/249277/UK_Solar_PV_Strategy_Part_1_Roadmap_to_a_Brighter_Future_08.10.pdf
} 
by fitting the data to an S-curve. Until then, the implications of changing $p$ and $q$ can be analysed. Hence, a variety of scenarios can be explored with differing levels of external and internal pressures.

More specifically, in Section 2, a travelling wave solution is derived that is dependent upon the equation parameters $p$ and $q$. In addition, the variation of these parameters and the effect this has on the adoption curves is investigated. Next, in Section 3, the geographic area of interest is outlined, as well, so to apply the FEM, the grid is constructed. Then, in Section 4, (1) is applied to this grid to simulate the spread of innovations and the results are depicted. Furthermore, innovation uptake at a household level is considered.

\section{The Model}

The innovation diffusion model proposed by Haynes et al. (1977) is now transformed into the Fisher equation. Then, the analysis related to the Fisher equation is used to predict adoption times as a function of $p$ and $q$.

To begin, let

$$
t^{*}=(p+q) t, \quad x^{*}=\sqrt{\frac{p+q}{k}} x, \quad y^{*}=\sqrt{\frac{p+q}{k}} y, \quad N=(1+p / q) u-p / q .
$$

As a result, (1) becomes

$$
\frac{\partial u}{\partial t^{*}}=u(1-u)+\frac{\partial^{2} u}{\partial x^{* 2}}+\frac{\partial^{2} u}{\partial y^{* 2}} .
$$

This is the Fisher equation in two spatial dimensions. Ablowitz and Zeppetella (1979) found an exact travelling wave solution to (3), which is

$$
u=\frac{1}{\left(1+\exp \left(\frac{1}{\sqrt{6}}\left(x^{*}-c t^{*}\right)\right)\right)^{2}},
$$

where $c=\frac{5}{\sqrt{6}}$ is the wave speed. This wave propagates along the $x^{*}$-axis $\left(u_{y^{*}} y^{*}=0\right)$ and is monotonically increasing with $t^{*}$, at fixed $x^{*}$. This solution type is a suitable model for household technology uptake since it is assumed here that a property will retain the acquired innovation. For instance, once a PV is installed at a residence, it is believed to be highly unlikely the system will be removed within the foreseeable future. Furthermore, from (2) and (4) it is evident that as time increases, $u, N \rightarrow 1$. Hence, $100 \%$ adoption everywhere is the upper limit, which means that all households will eventually obtain the innovation using this model.

Next, by rotating the reference frame of (4), Tyson and Brazhnik (2000) identified the family of solutions

$$
u=\frac{1}{\left(1+\exp \left(\frac{1}{\sqrt{6}}\left(x^{*} \sin \theta+y^{*} \cos \theta-c t^{*}\right)\right)\right)^{2}},
$$

such that the waves propagate at some angle $\theta$ from the $x^{*}$-axis. So, if our focus is how (5) evolves with increasing $t^{*}$ at a fixed location, then let us set

$$
u=\frac{1}{\left(1+\exp \left(\frac{1}{\sqrt{6}}\left(\tilde{z}-c t^{*}\right)\right)\right)^{2}},
$$

where $\tilde{z}$ is some constant. This solution is plotted in Figure 1, top left with $\tilde{z}=0(\tilde{z} \neq 0$ will simply shift the solution right or left in this figure). 
Re-writing (5) in terms of the adopter ratio, the following travelling wave solution to (1) is attained

$$
N=\left(1+\frac{p}{q}\right) \frac{1}{\left(1+\exp \left(\frac{1}{\sqrt{6}}\left(\sqrt{\frac{p+q}{k}} x \sin \theta+\sqrt{\frac{p+q}{k}} y \cos \theta-c(p+q) t\right)\right)\right)^{2}}-\frac{p}{q}
$$

This solution has the wave speed $c \sqrt{k(p+q)}$ in $(x, y, t)$ space and therefore, when $p$ and $q$ are small, progression is slow. From (7), it is apparent that at some fixed location, the growth of $N$ as a function of time is independent of the diffusion coefficient $k$. As well, $k$ can be removed from the governing equation (1) with the change of variables $\left(x^{\prime}, y^{\prime}\right)=(x / \sqrt{k}, y / \sqrt{k})$, and therefore, varying $\mathrm{k}$ simply re-scales the spatial domain. Hence, given that the effects of $p$ and $q$ on the adoption times are the main focus here, we set $k=1$ without any loss of generality for the remainder of this paper.

Now suppose we are interested in the time taken for $\mathrm{N}$ to increase to 0.99 , at a fixed location and beginning at zero, then, it is necessary to calculate

$$
\Delta t=t_{2}-t_{1}=\frac{6}{5(p+q)} \log \left|\frac{\sqrt{q / p+1}-1}{\sqrt{\frac{1+p / q}{0.99+p / q}}-1}\right|
$$

where $N\left(t_{1}\right)=0$ and $N\left(t_{2}\right)=0.99$. Therefore, specifying $p$ and $q$ determines $\Delta t$, the time domain of interest.

Next, let $u\left(t_{1}^{*}=(p+q) t_{1}\right)=u_{1}=1 /((q / p)+1)$ and $u\left(t_{2}^{*}=(p+q) t_{2}\right)=u_{2}=(0.99+(p / q)) /(1+(p / q))$ when $N=0$ and $N=0.99$ respectively, where $\Delta t^{*}=t_{2}^{*}-t_{1}^{*}=(p+q) \Delta t$ and the solution window is defined as $u \in\left[u_{1}, u_{2}\right]$. Therefore, varying $p$ and $q$ changes this solution domain. As an example, see Figure 1 , top right, where the solution windows for the two cases $p=0.0001, q=0.1$ and $p=0.01, q=0.1$ are depicted. This figure demonstrates that if $p / q$ is very small such that the imitation (internal) effect dominates, the estimated uptake is sluggish in the beginning and further time is needed before there is any evident rise in the overall adoption ratio. This behaviour is due to uptake being initially very localised. In contrast, when $p / q$ is greater and the innovation coefficient is amplified, adoptions are almost immediate. Uptake is now no longer at a local level but the process instead occurs more uniformly across the entire population. In Section 4 , the influences of $p$ and $q$ on our local electricity network are further explored.

Due to (8), a solution parameter space can be defined in terms of $p, q$ and $\Delta t$, which is shown in Figure 1 , bottom left. This figure reveals that the adoption times decrease when $p$ and $q$ are increased, which is expected. Note that this depicted parameter space is unaffected by the choice of $k$. Here, $\Delta t=30$ years is used as a hypothetical case, which means that overall at least $99 \%$ of our sample population will have adopted the technology in 30 years. We will study three scenarios corresponding to $\Delta t=30$ years that differ in their levels of internal and external pressures. These are $(a) p=0.001, q=0.325$, (b) $p=0.0001, q=0.376$ and (c) $p=0.00001, q=0.425$. The adoption trajectories for these three cases are shown in Figure 1, bottom right, defined by (7). All these curves converge as $t \rightarrow 30$ years.

\section{The Grid}

An analysis of a local electricity network in Bracknell, UK is now undertaken, which comprises of 9484 households and 249 feeders. Every household is connected to a certain feeder, where electricity is supplied to 

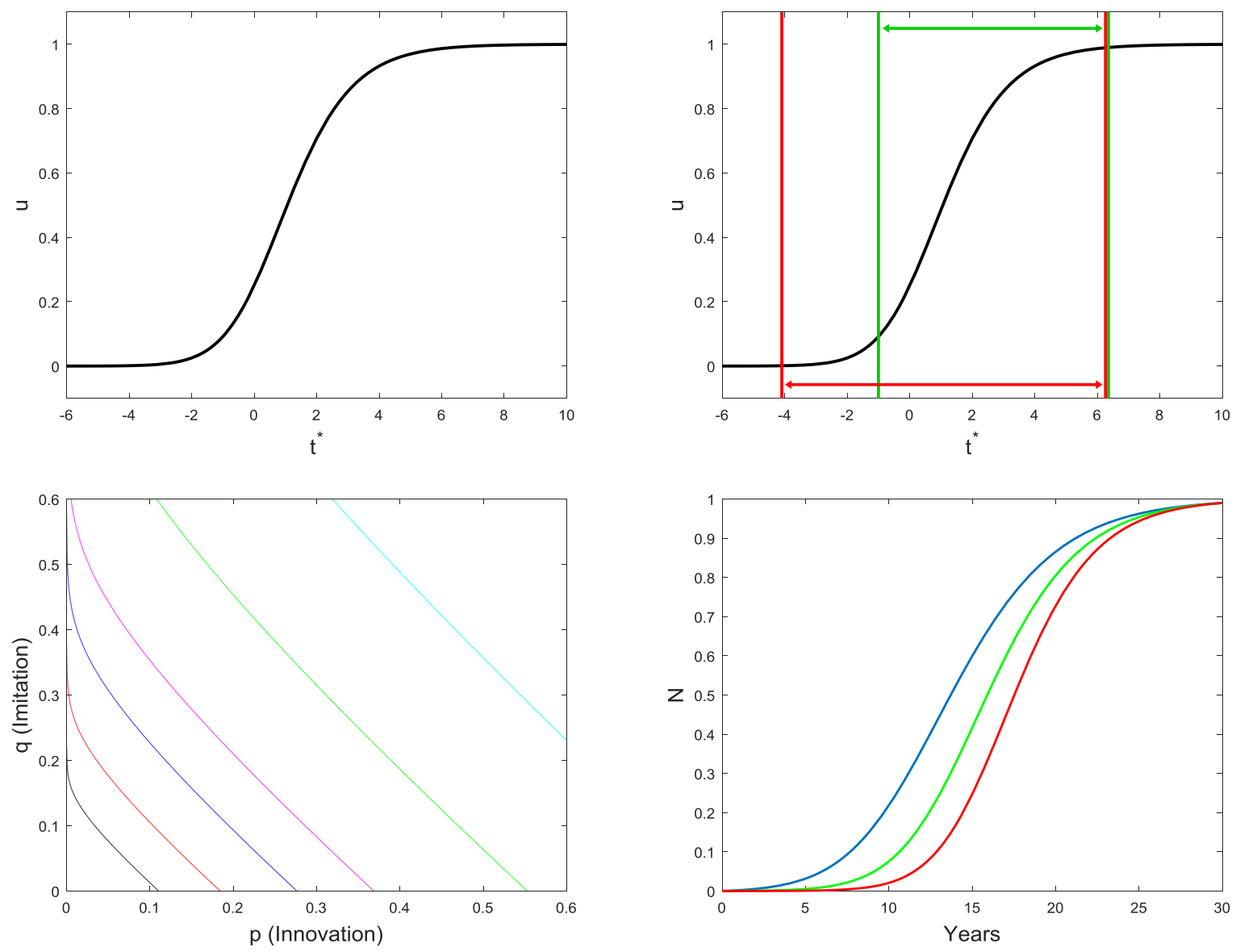

Figure 1: Top Left: Plot of $u$ defined by (6) when $\tilde{z}=0$. Top Right: Solution window for $p=0.0001, q=0.1$ (red) and $p=0.01, q=0.1$ (green). Bottom Left: Time required to achieve at least $99 \%$ uptake everywhere as a function of $p$ (innovation coefficient) and $q$ (imitation coefficient), refer to (8); black: $\Delta t=50$ years, red: $\Delta t=30$ years, blue: $\Delta t=20$ years, purple: $\Delta t=15$ years, green: $\Delta t=10$ years, light blue: $\Delta t=7$ years. Bottom Right: Innovation uptake curves for $p=0.001, q=0.325$ (blue), $p=0.0001, q=0.376$ (green) and $p=0.00001, q=0.425$ (red) defined by (7). 

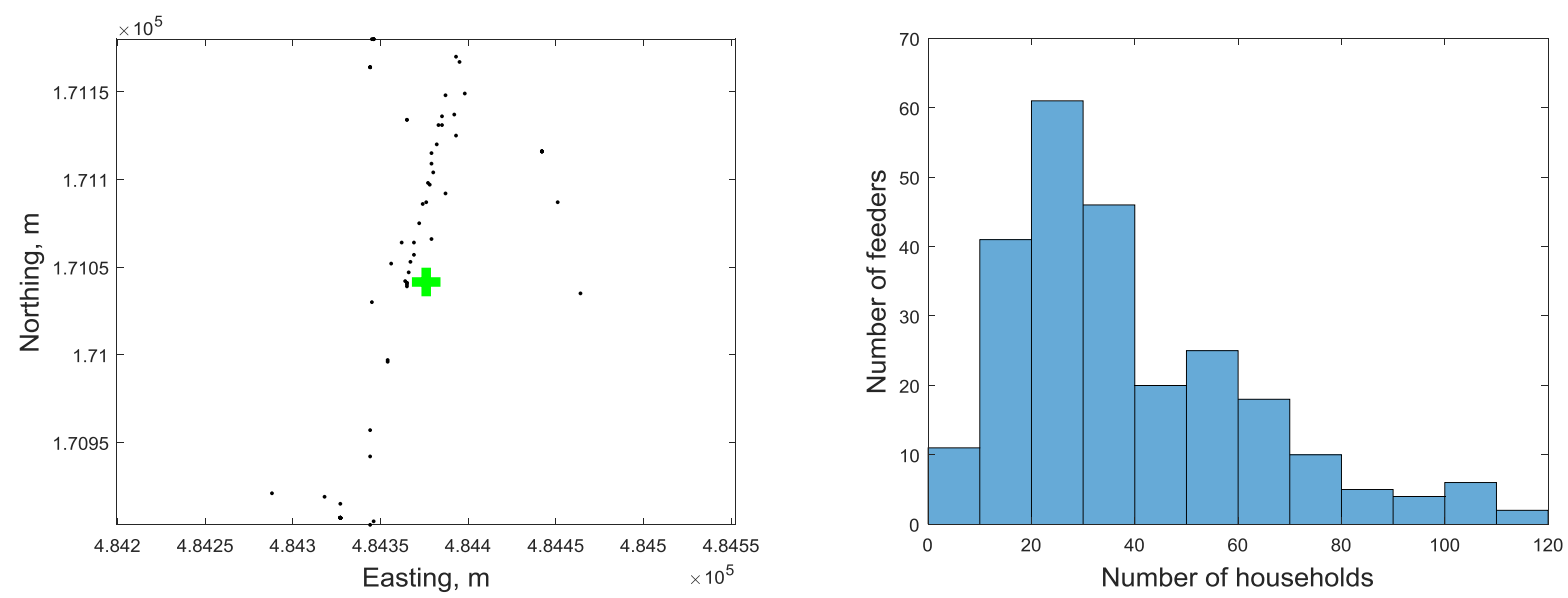

Figure 2: Left: Determining the feeder co-ordinates (green cross) for a feeder with 84 households (black dots). Right: The distribution of households amongst the 249 feeders.

a home along its assigned feeder. Therefore, these assignments represent spatial clusters of households. The household locations and feeder allocations used in this paper are based upon real data.

Here, (1) is applied to model innovation diffusion amongst the feeders using the FEM, where the imitation and innovation coefficients are varied. As a result, we can assess the effects of internal and external pressures at a local level. More specifically, adoption trajectories will be determined for each feeder, which is the expected uptake at a fixed location as a function of time. These projections are important measures for network planners as they indicate the potential, additional demand at specific network sites. This anticipated extra load can be accounted for at the design stage so that issues such as localised network damage or disconnections can be avoided when the network experiences periods of high demand.

We are concerned with the uptake of LCTs, including electric vehicles (EVs) and photovoltaics (PVs) since these are expected to be employed throughout the UK and to have a significant impact upon the existing electricity network. Haynes' equation, (1), is chosen to estimate the spread of LCTs across our area of interest. This is due to the characteristics of its solutions already discussed, which are monotonicity and convergence that make this model suitable for predicting LCT adoption. For an example of (1) being used in a similar context see Shinohara and Okuda (2009), where the uptake of hybrid cars in Japan was modelled.

Firstly, our finite element grid needs to be generated. The feeder locations in $(x, y)$ space are used to determine this, where the units for $x$ and $y$ are easting (meters) and northing (meters) respectively. The $(x, y)$ co-ordinate of a feeder corresponds to the midpoint of all its households in the $x$ and $y$ directions. In Figure 2 , left, an example is given of this calculation for a feeder with 84 households. It should be noted that the number of households assigned to each feeder varies somewhat. Refer to Figure 2, right, which demonstrates the distribution of households amongst the 249 feeders.

By examining the feeder locations it is evident that they too are clustered into a number of distinct regions. So, we assign these points to separate zones. To ascertain appropriate groupings of the feeders, a circle of radius $r=305$ meters is formed around each feeder location. These circles are then used to signify the approximate area that feeders can influence or be influenced by other feeders. This area can be modified 
by changing $r$. Next, a region is defined by drawing a boundary around the circles that overlap. Using this method, six distinct shapes are created and when combined, they form our grid. See Figure 3 for depictions of the six separate regions. Then, in Figure 4, left, the union of these shapes is illustrated, which represents the grid. As well, a triangular element mesh has been created and portrayed in Figure 4, right. Open boundaries exist when shapes overlap, otherwise each region is a closed system. The model (1) is applied to this grid using the FEM.

To validate the analysis detailed in Section 2, the numerical result found using MATLAB's pde solver is compared to (7), where the imposed initial condition is $N(t=0)=0$ everywhere (refer to Figure 4 for depictions of the solution domain). The comparisons for the three cases $(a),(b)$ and $(c)$ are given in Figure 5 , where a good agreement is observed. Note that since $N=0$ everywhere at $t=0$, then $N$ remains spatially uniform for all $t>0$. Thus, the solution curves depicted in Figure 5 are the same for all $(x, y)$.

\subsection{The Initial Condition}

The initial condition for the simulations needs to be determined. We know the location of 52 households with PVs installed and therefore, these initial sources of innovation will be included in the model. However, this is a small number when considering there are 9484 households in total. Therefore, an additional 100 properties have been randomly assigned a PV for illustrative purposes. Hence, 152 households are used as initial sources for modelling PV adoption throughout our local network.

The focus here is now the adoption of PVs within Bracknell since PV properties are known. Although, the technique presented in this paper can be used to model other LCT types, such as EVs, assuming that the adoption behaviour is dependent upon internal and external influences. For PV uptake, it is expected that the imitation effect will dominate as the spatial clustering of PV properties is a known phenomenon. For example, Kwan (2012) demonstrated that across the United States (especially in California), a correlation between zip code and a higher concentration of PV households existed. Therefore, clusters of households, like a neighbourhood, do influence one another so that spatial groupings of PV adopters form. This can be attributed to the social interactions of neighbours or the observations of neighbour behaviour. Other potential factors to consider are that neighbours generally have similar attitudes, property sizes and incomes.

A source at the feeder location $\left(x_{i}, y_{i}\right)$ is defined by

$$
s_{i}=a_{i} \operatorname{sech}\left(\beta\left(\sqrt{\left(x-x_{i}\right)^{2}+\left(y-y_{i}\right)^{2}}\right)\right)^{2},
$$

where $a_{i}$ is the accumulative feeder adoption ratio at $t=0$ and $N(x, y, t=0)=\sum_{i=1}^{152} s_{i}$. Now, as $\beta$ decreases, the width of this solution increases. Therefore, we have made $\beta$ dependent upon the number of households along the feeder, choosing $\beta=0.006,0.012,0.018$ to correspond to the feeder populations $0-40$ households, $40-80$ households and $80-120$ households respectively. These values of $\beta$ are applied so to reflect the approximate geographical size of the feeder. However, this is a model parameter that can be varied. See Figure 6 for an example of a source located at $(484000,171000)$ with $a_{i}=1$ and $\beta=0.018$ (left), $\beta=0.012$ (middle) and $\beta=0.006$ (right).

Thus, using (9) to define sources at the 152 highlighted properties, the initial condition for our model is established. This solution, $N(x, y, t=0)$, is depicted in Figure 7 . On the left, only the known PV locations are displayed and on the right, the combination of the known PV and the randomly assigned households is 

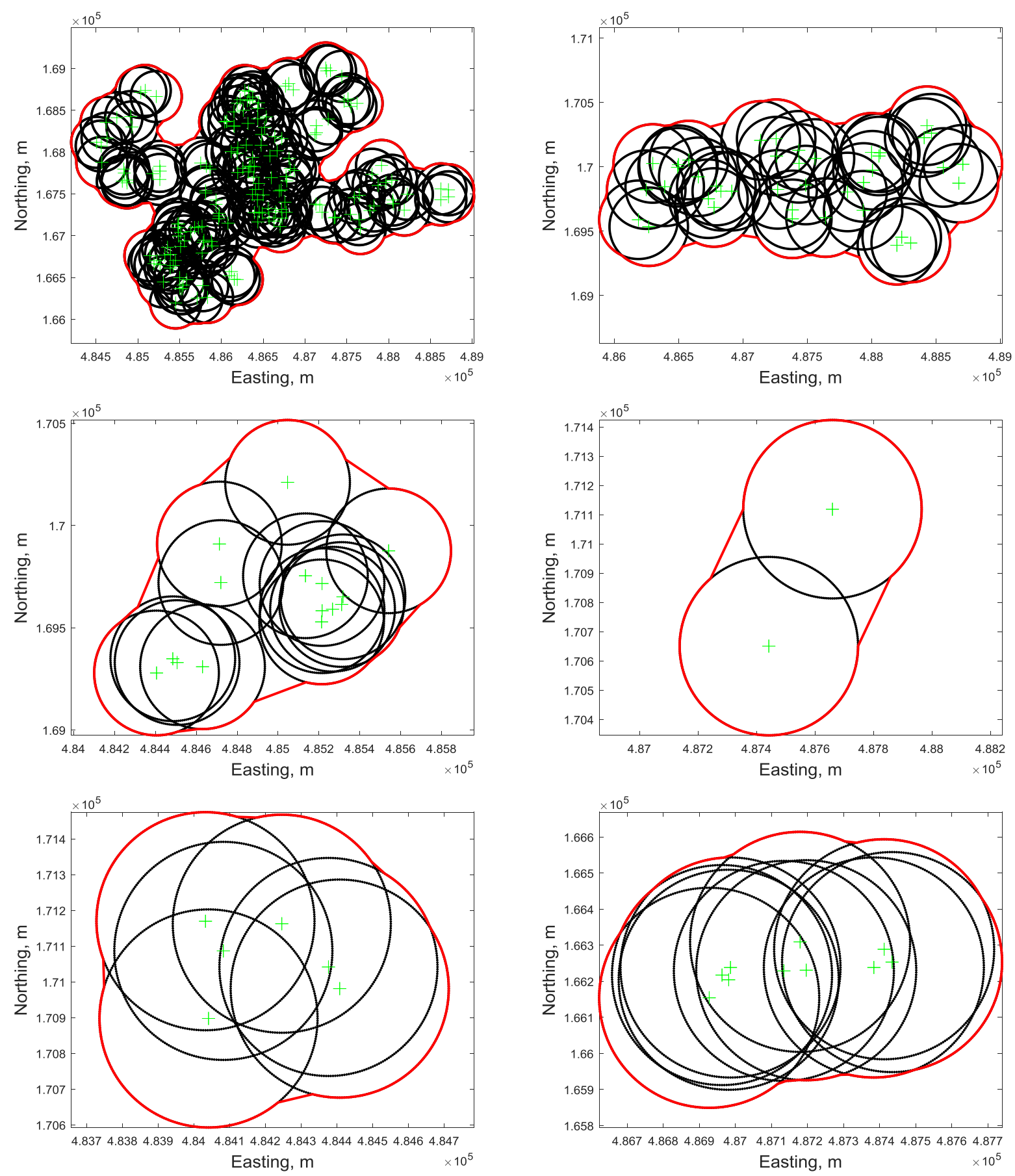

Figure 3: The six distinct shapes that represent clusters of feeders, where the green crosses correspond to feeder locations and the red outline defines the shape. The black circles centred at the feeder locations have a radius of $r=305 \mathrm{~m}$. The union of these six red shapes is depicted in Figure 4. 

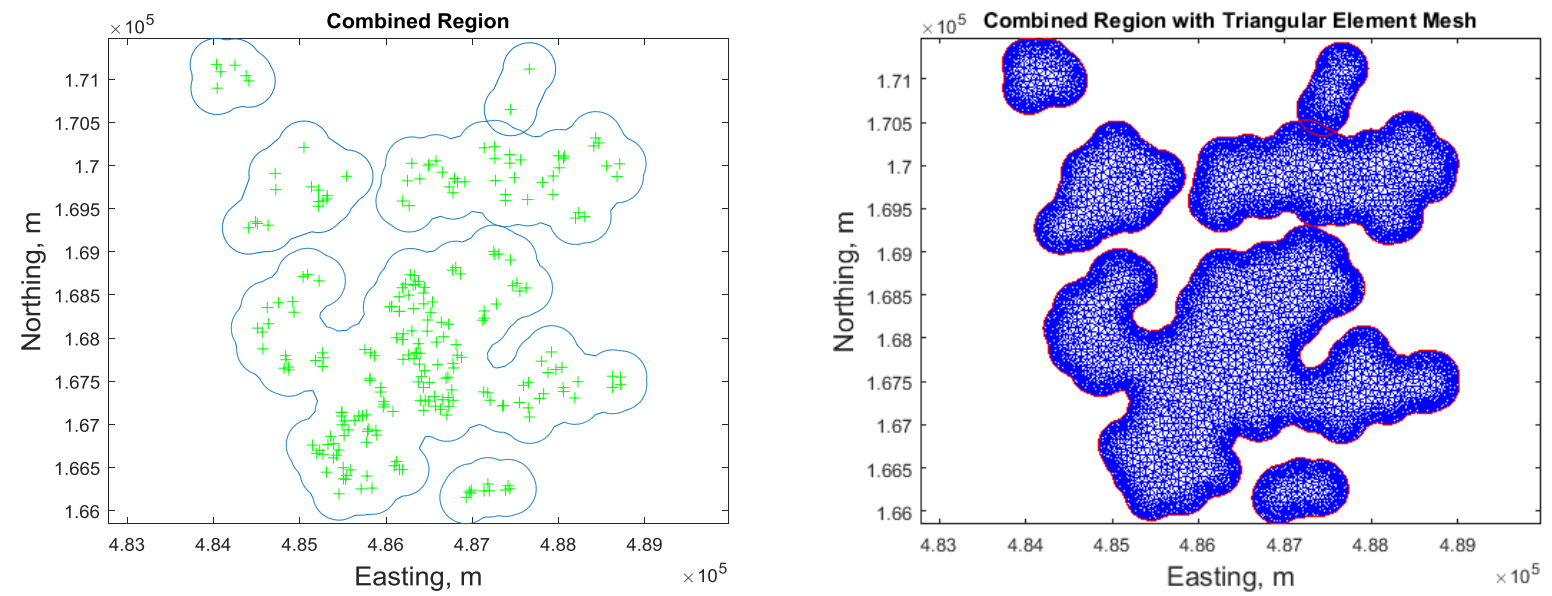

Figure 4: Left: The union of the six shapes shown in Figure 3 with the feeder locations indicated by green crosses. Right: The union of the six shapes shown in Figure 3 with a triangular element mesh applied. This shaded area defines our FEM grid.
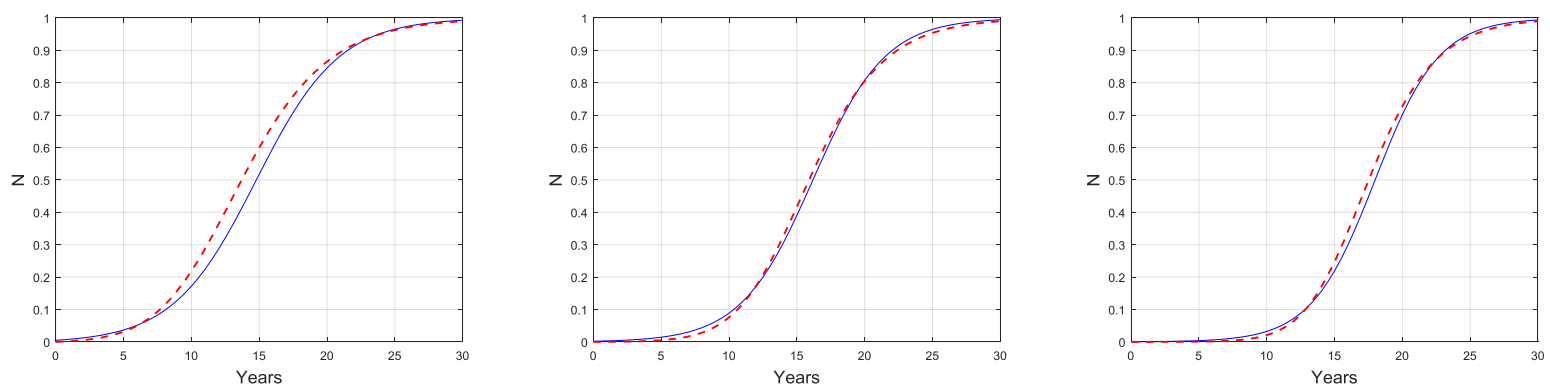

Figure 5: Comparing the numerical result (blue) with the analytical solution (red dashed) when $N(t=0)=0$ everywhere, where $p=0.001, q=0.325$ (left), $p=0.0001, q=0.376$ (middle) and $p=0.00001, q=0.425$ (right).
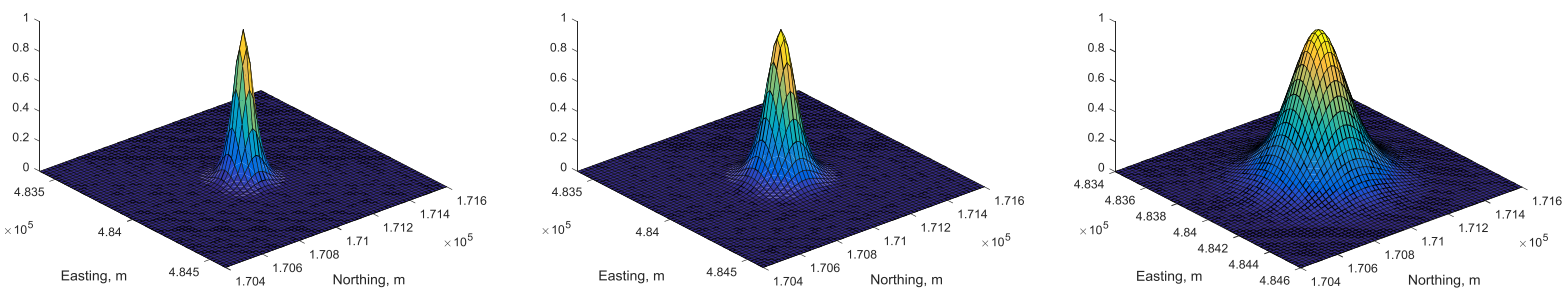

Figure 6: Sources at the feeder location (484000,171000) with 0 - 40 households (left), 40 - 80 households (middle), $80-120$ households (right). Grid size: 1200m by $1200 \mathrm{~m}$. 

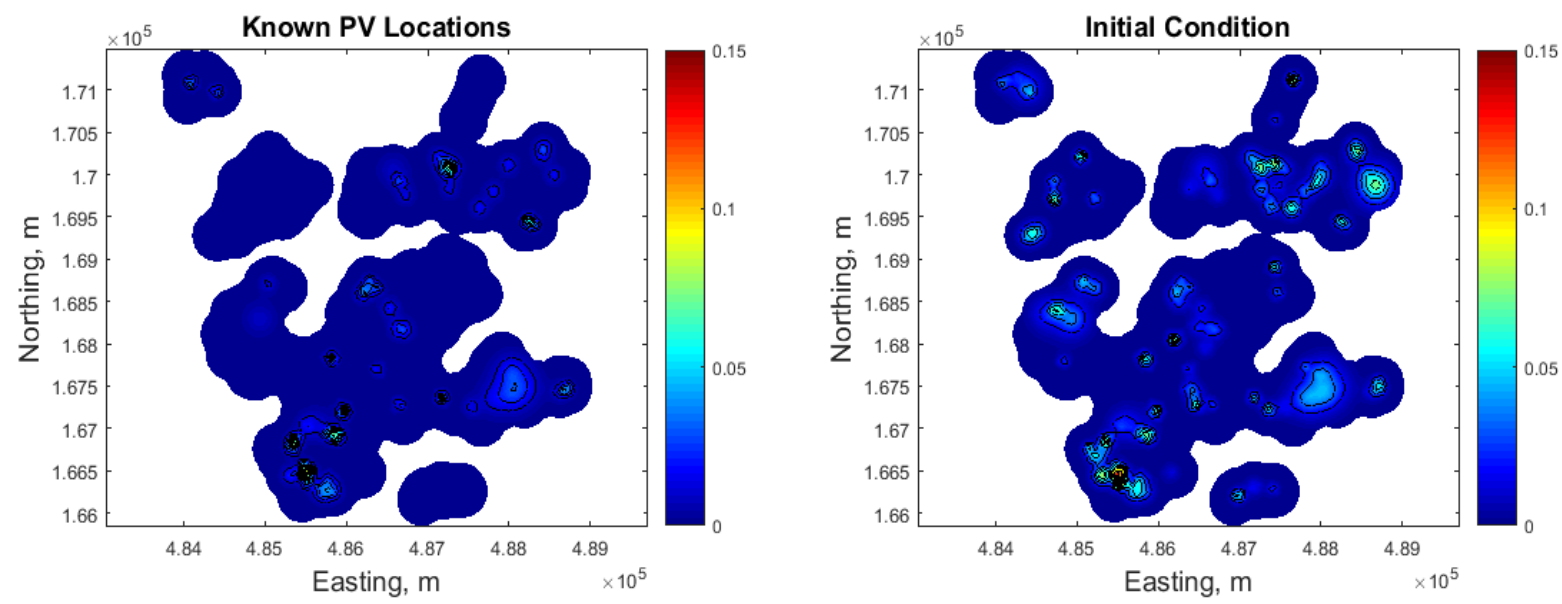

Figure 7: Defining the initial condition using (9). Left: Known PV locations. Right: Known PV locations and the 100 randomly assigned $P V$ households. The legend indicates the adoption ratio, $N$.

shown. This proposed initial condition is used here for illustrative purposes. Note that the choice of the initial condition will have a large impact upon the model predictions. Therefore, when further data becomes available, this condition should be modified to reflect real-life initial uptake behaviour.

\section{Simulations}

Finite element modelling was performed with MATLAB's pde solver for the scenarios $(a)-(c)$, where the initial condition depicted in Figure 7, right was applied. As already stated, these cases represent $\Delta t=30$ years and therefore, all results from the simulations will converge to approximately $99 \%$ as $t \rightarrow 30$ years.

In Figures 8 and 9, the results at $t=5,10,15,20$ years are displayed. As expected, when the imitation coefficient is much larger than the innovation effect, the adoption clusters surrounding the feeder locations become notably more intense with increasing time. For instance, comparing the maps at $t=10$ years for cases $(a)-(c)$, it is apparent that case $(c)$ exhibits much greater adoption numbers in the areas around the feeders with sources. As $t$ becomes larger, these clusters expand and amplify. However, it should be noted that the background, overall adoption ratio (this follows the trends given in Figure 5) is evidently greater, in particular at $t=15,20$ years, when the innovation influence is increased. Consequently, the contrast between the adoption clusters and the background level is far more apparent for case $(c)$. These observations are expected since $q$ will have a localised effect (household adoptions resulting from neighbour activity), whereas $p$ has an overall impact (external factors encouraging adoptions).

Next, comparisons of uptake trajectories at different fixed feeder locations are given in Figure 10. On the left, the positioning of the feeder is indicated by a white cross on the grid, and on the right, the adoption ratio as a function of time is displayed for all three cases. Technology clusters form here since these highlighted feeders have non-zero initial conditions, as well, they are influenced by neighbouring feeders with sources. The adoption curves reveal that the rate of uptake at these particular feeders is greater when the magnitude of $p$ decreases and $q$ increases. The exhibited behaviour is again expected since $q$ promotes the spread of 
technologies at a local level.

\subsection{Uptake at a household level}

We have predicted adoption trajectories at the feeder locations based on the initial condition portrayed by Figure 7, right. These curves reveal a macroscopic viewpoint of the adoption behaviour over time, although, the adoption activity at a household level (microscopic) has not yet been discussed. To estimate this, the feeder curves obtained using the FEM can guide the household selection. More precisely, the number of adoptions at a particular feeder and time is specified by its trajectory (see Figure 10, right for depictions of some feeder curves). This combination of micro-scale and macro-scale models corresponds to a hybrid approach. For an example of a hybrid model, see Swinerd and McNaught (2014), where innovation diffusion across the world was simulated. Firstly, the adoption timings of different country groupings was estimated with the theory of Bass (1969). Then, at the next level, an agent-based model determined the actions of individual countries, where the impact of neighbouring countries' choices was taken into account.

An example of household uptake after eight years is depicted for case $(a)$ and $(c)$ in Figure 11, where the properties were randomly selected such that the number of adoptions reflect the FEM results at $t=8$ years. The top and middle panels reveal PV household density plots and the FEM results respectively. As well, the overall adoption numbers are given as a function of time along the bottom panel of Figure 11. A more sophisticated technique can be applied to assign technologies at a household level by using an agent-based approach, where certain households are favoured due to individual characteristics. Refer to Hattam and Greetham (2017) where an agent-based model was applied to a subset of the geographic area examined here. This study forecasted electric vehicle and photovoltaic uptake using socio-demographic information to make certain households more likely of LCT adoption.

Upon close inspection of the top panel of Figure 11, it becomes apparent that more densely populated and compact clusters develop when $p$ is reduced and $q$ is increased, which is expected. Interestingly, the number of household adoptions after eight years is 2688 and 3382 for case $(a)$ and case $(c)$ respectively. Moreover, the overall adoption curves reveal that the case $(c)$ parameters encourage increased household uptake numbers for approximately $t \in(0,30)$, where both trends converge as $t \rightarrow 30$ years. This can be attributed to the feeders being closely spaced and the intense adoption hot-spots that form as a result of the FEM.

\section{Conclusion}

The diffusion of innovations over time and space was explored using the model (1), which was initially proposed by Haynes et al. (1977). More specifically, we concentrated on the spread of photovoltaics amongst 9484 households that were linked to an electricity network within Bracknell, UK comprising of 249 feeders. All households were allocated to a particular feeder, where the feeder co-ordinates were defined as the midpoint of all its connected properties. These co-ordinates then allowed us to generate a grid that represented the local network under investigation. Once this solution domain was outlined, the FEM was utilised to forecast PV uptake with (1), which was dependent upon the coefficients $p$ (innovation) and $q$ (imitation). By then relating these parameters to the well-known Fisher travelling wave solution derived by Ablowitz and Zeppetella (1979), estimates for the adoption times were found as a function of $p$ and $q$. However, since currently insufficient 

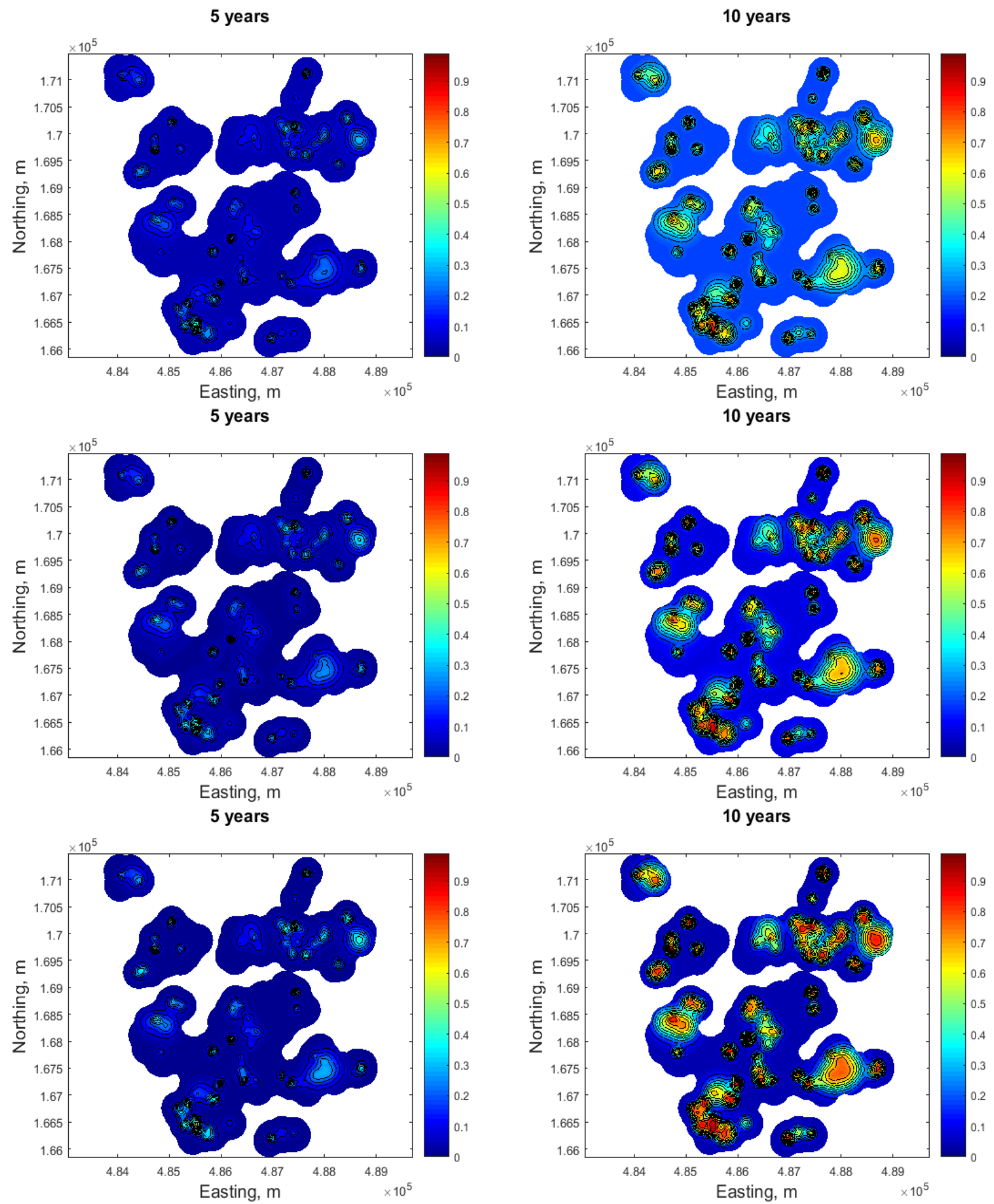

Figure 8: Innovation diffusion simulations at $t=5$ years (left) and $t=10$ years (right) for case $(a): p=0.001, q=0.325$ (top), case (b): $p=0.0001, q=0.376$ (middle) and case (c): $p=0.00001, q=0.425$ (bottom). The legend indicates the adoption ratio, $N$. 

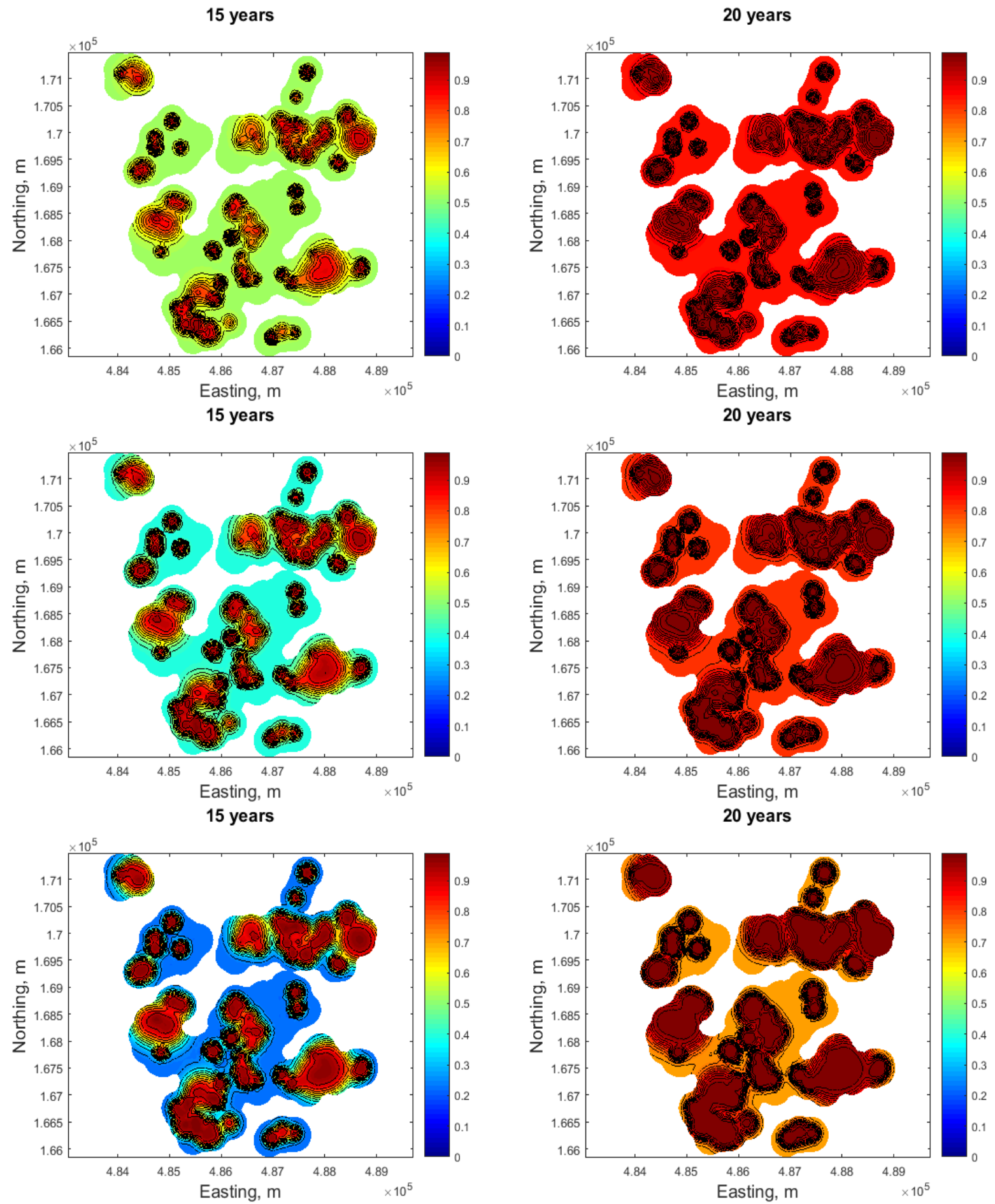

Figure 9: Innovation diffusion simulations at $t=15$ years (left) and $t=20$ years (right) for case $(a): p=0.001, q=0.325$ (top), case (b): $p=0.0001, q=0.376$ (middle) and case (c): $p=0.00001, q=0.425$ (bottom). The legend indicates the adoption ratio, $N$. 

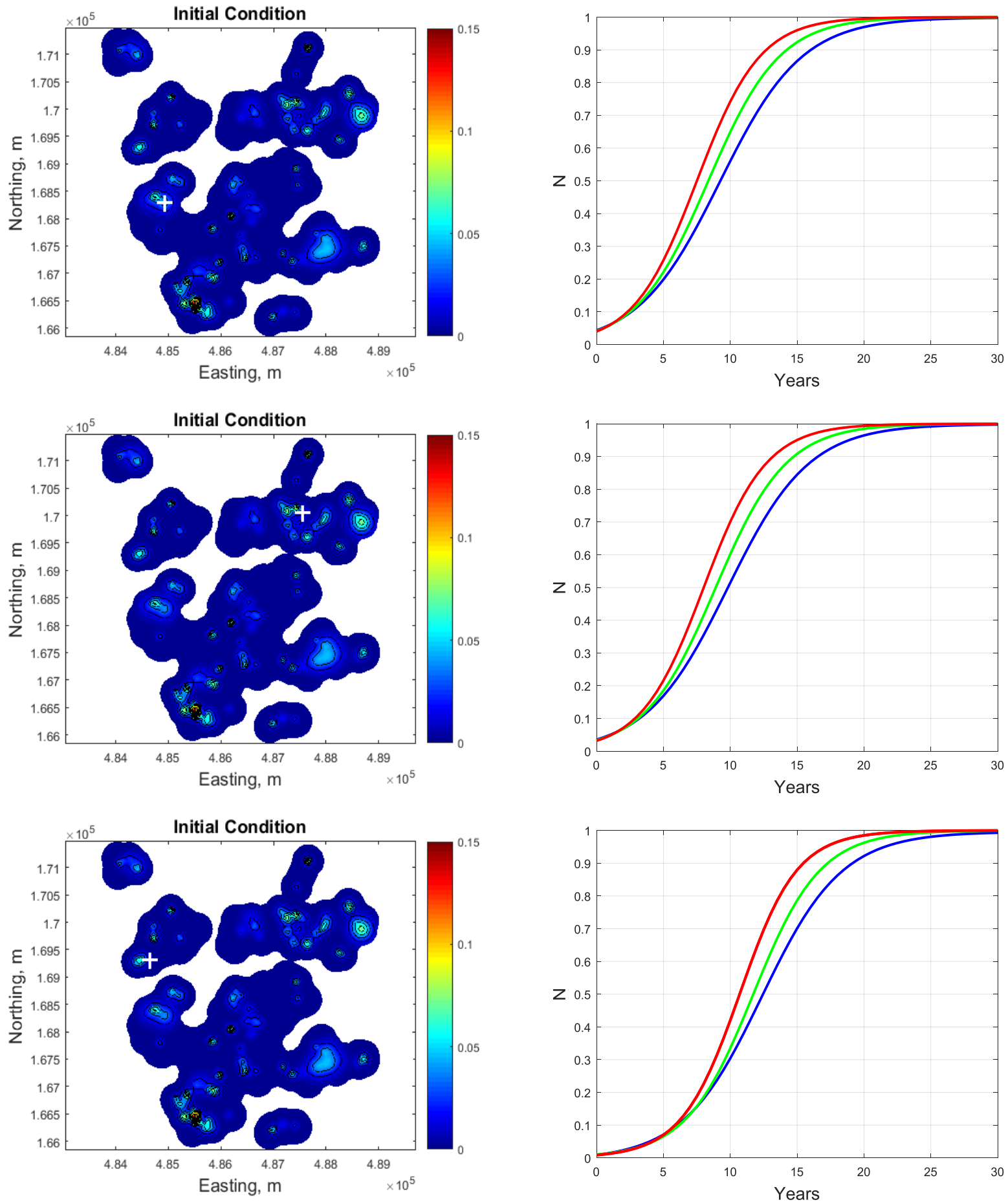

Figure 10: Innovation adoption curves at select feeders, where $p=0.001, q=0.325$ (blue), $p=0.0001, q=0.376$ (green) and $p=0.00001, q=0.425$ (red). Top: Feeder location (484930,168300). Middle: Feeder location $(487560,170060)$. Bottom: Feeder location $(484650,169310)$. Left: Feeder location indicated by the white cross on the grid. Right: Innovation adoption curves, $N(t)$. 

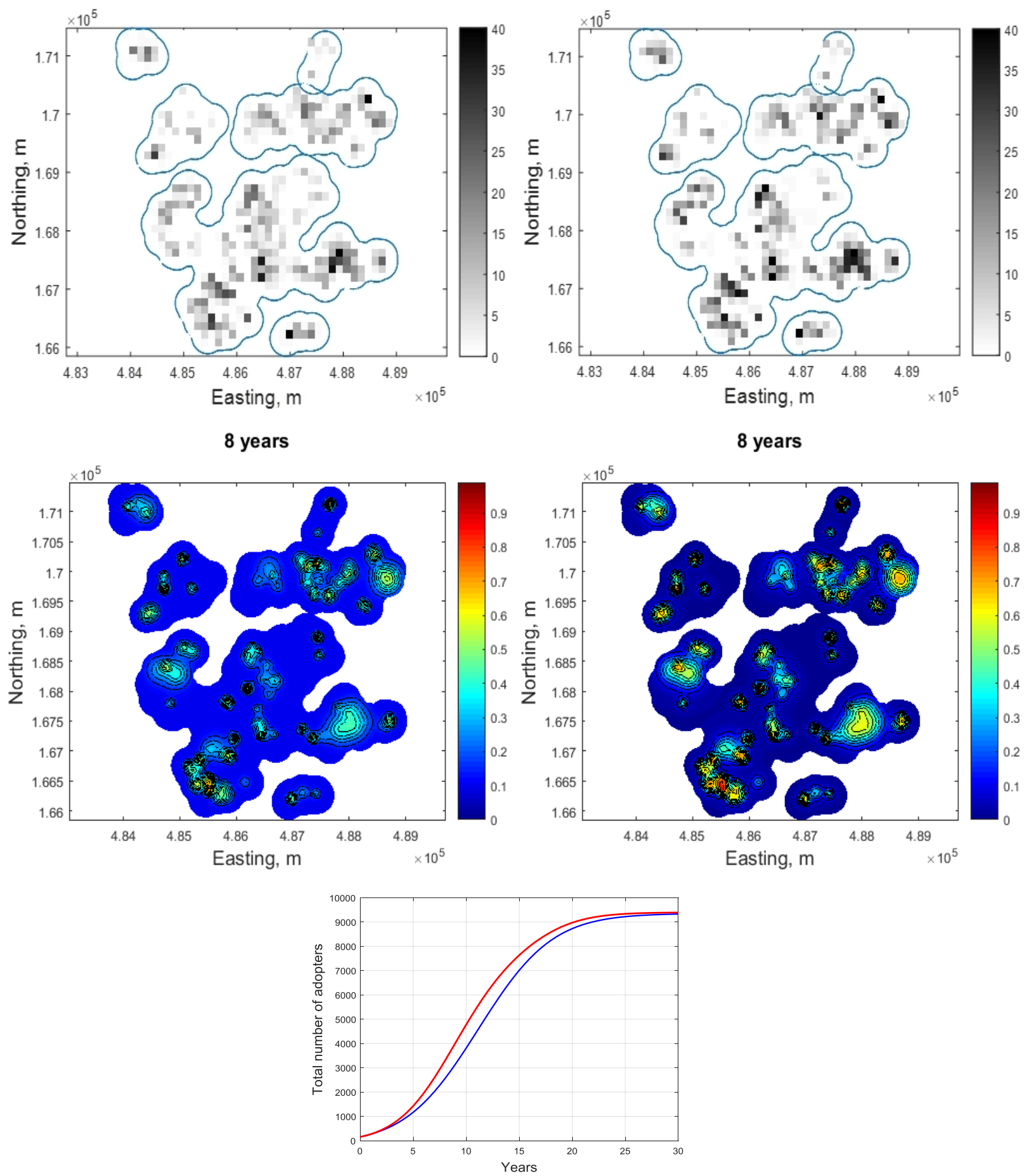

Figure 11: Household level uptake. Top Left: Data density plot that represents 2688 PV households for $p=0.001$, $q=0.325$. The legend indicates the number of PV households at this location. Top Right: Data density plot that represents $3382 P V$ households for $p=0.00001, q=0.425$. The legend indicates the number of $P V$ households at this location. Middle: Innovation diffusion maps at $t=8$ years, for $p=0.001, q=0.325$ (left) and for $p=0.00001, q=0.425$ (right). The legend indicates the adoption ratio, N. Bottom: Overall number of adopters as a function of time, where the red and blue curve signify $p=0.00001, q=0.425$ and $p=0.001, q=0.325$ respectively. 
information is available to select accurate initial model conditions and imitation/innovation coefficients, various illustrative cases were instead presented. Although future work will involve acquiring additional reallife data to incorporate into the model. Here, differing values of $p$ and $q$ were applied that corresponded to a consistent adoption time of $\Delta t=30$ years. As a result, the impact of both these factors on our local network was assessed over a set time frame. Next, the results of our simulations were detailed, where more obvious and intense innovation clusters developed over time when the imitation (internal) effects dominated considerably. In addition, feeder uptake curves were depicted, which were projections for the adoption ratio at some feeder as a function of time. These measures revealed that adoption rates rose at feeders with sources if the imitation influences were increased. Moreover, the feeder curves provided an overall, aggregate understanding of the innovation diffusion process. These projections are potentially very informative estimates for network planners since the predicted increase in load can be accounted for now so to avoid future network issues. Finally, modelling technology uptake at a household level was addressed, where houses were randomly assigned PVs by following the feeder trends, merging macroscopic and microscopic techniques. The results presented here suggested that more households installed a PV if the imitation effect outweighed innovation considerably, which was due mainly to the closely spaced feeder locations. Thus, a model has been outlined to estimate the spread of technologies and the impact this will have on a local electricity network, where internal and external influences were considered. Once further information becomes available so that the calibration can be improved, this model will then become an important predictive tool for the planners of the network.

\section{Acknowledgment}

This work was carried out with the support of Scottish and Southern Electricity Networks through the New Thames Valley Vision Project (SSET203 New Thames Valley Vision) funded by the Low Carbon Network Fund established by Ofgem.

\section{References}

M.J. Ablowitz and A. Zeppetella. Explicit solutions of Fisher's equation for a special wave speed. Bulletin of Mathematical Biology, 41(6):835-840, 1979.

F.M. Bass. New product growth for model consumer durables. Management Science, 15(5):215-227, 1969.

G.W.A. Constable, T. Rogers, A.J. McKane, and C.E. Tarnita. Demographic noise can reverse the direction of deterministic selection. Proceedings of the National Academy of Sciences, 113(32):E4745-E4754, 2016.

R.A. Fisher. The wave of advance of advantageous genes. Ann. of Eugenics, 7:355-369, 1937.

L. Hattam and D. Vukadinovic Greetham. Green neighbourhoods in low voltage networks: measuring impact of electric vehicles and photovoltaics on load profiles. Journal of Modern Power Systems and Clean Energy, 5(1):105-116, 2017. 
K.E. Haynes, V. Mahajan, and G.M. White. Innovation diffusion: A deterministic model of space-time integration with physical analog. Socio-Economic Planning Sciences, 11(1):25-29, 1977.

E. Karakaya. Finite Element Method for forecasting the diffusion of photovoltaic systems: Why and how? Applied Energy, 163:464-475, 2016.

C.L. Kwan. Influence of local environmental, social, economic and political variables on the spatial distribution of residential solar PV arrays across the United States. Energy Policy, 47:332-344, 2012.

A. Masini and P. Frankl. Forecasting the diffusion of photovoltaic systems in southern Europe. Technological Forecasting and Social Change, 70(1):39-65, 2003.

C. Michalakelis and T. Sphicopoulos. A population dependent diffusion model with a stochastic extension. International Journal of Forecasting, 28(3):587-606, 2012.

R.L. Morrill. The shape of diffusion in space and time. Economic Geography, 46:259-268, 1970.

E.M. Rogers. Diffusion of Innovations. The Free Press, New York, 1962.

K. Shinohara and H. Okuda. Dynamic innovation diffusion modelling. Computational Economics, 35(1):51, 2009.

J.R. Snape. Spatial and temporal characteristics of PV adoption in the UK and their implications for the smart grid. Energies, 9(3), 2016.

C. Swinerd and K.R. McNaught. Simulating the diffusion of technological innovation with an integrated hybrid agent-based system dynamics model. Journal of Simulation, 8(3):231-240, 2014.

J.J. Tyson and P.K. Brazhnik. On traveling wave solutions of Fisher's equation in two spatial dimensions. SIAM Journal on Applied Mathematics, 60(2):371-391, 2000. 